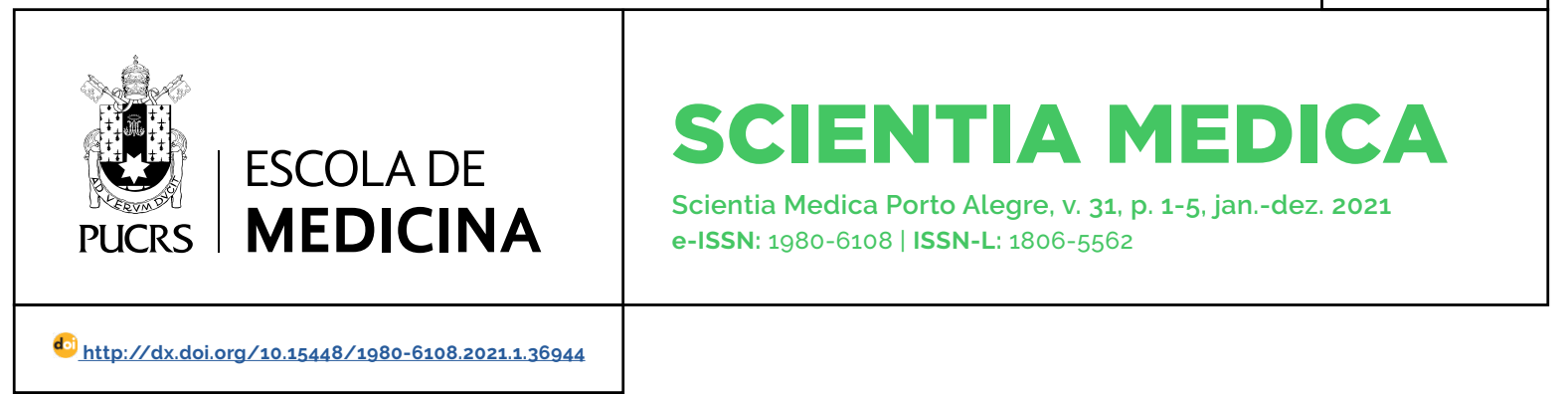

SEÇÃO: EDUCAÇÃO EM CIÊNCIAS DA SAÚDE

\title{
Anatomia Radiológica Vascular e desenvolvimento de plataformas de ensino interativo
}

\author{
Vascular Radiological Anatomy and the development of interactive teaching platforms
}

\section{Gustavo Bittencourt Camilo ${ }^{1}$ orcid.org/0000-0001-7387-8381 gustavoscamilo@hotmail.com}

José Otávio Guedes Junqueira ${ }^{2}$ orcid.org/0000-0002-8188-2900 jose.otavio@ufjf.edu.br

Henrique Guilherme de Castro Teixeira $^{2}$

orcid.org/0000-0001-9082-8925

hgcteixeira@gmail.com

Alice Belleigoli Resende ${ }^{2}$

orcid.org/0000-0002-2584-2496

alice.resende@ufjf.edu.br

\section{André Gustavo Fernandes de} Oliveira $^{2}$

orcid.org/0000-0001-8773-0273

agfo79@gmail.com

Pedro de Freitas Batista Mendes orcid.org/0000-0001-6468-3515 pedrofreitas097@gmail.com

Gabriela Cumani Toledo ${ }^{3}$ orcid.org/0000-0003-2654-8894 gabrielacumanig@gmail.com

\section{Matheus Cruz Ferraro ${ }^{3}$}

orcid.org/0000-0002-9900-8453 matheuscruzferraro@hotmail.com

Thais Bandeira de Oliveira Junqueira 4

orcid.org/0000-0003-1322-0216

thaisbandeira94@gmail.com

Bruna Bandeira de Oliveira Junqueira ${ }^{5}$

orcid.org/0000-0003-0989-033X bruninha.bandeirag8@gmail.com

Recebido em: 28/1/2020.

Aprovado em: 12/12/2020

Publicado em: 26/4/2021

\section{(c) (i)}

Artigo está licenciado sob forma de uma licença Creative Commons Atribuicão 4.0 Internacional.
Resumo: Anatomia e Radiologia são disciplinas amplamente presentes nas grades curriculares de cursos da área da saúde. A interseção entre a Anatomia Radiológica e as tecnologias recentes, como plataformas de ensino interativo, caracteriza-se como uma tendência a ser seguida na esfera educacional, sendo as implicações desse processo ainda não muito debatidas. O Departamento de Anatomia da Universidade Federal de Juiz de Fora iniciou o desenvolvimento de um atlas de anatomia vascular radiológica, o qual apresenta versões impressa e interativa. Possui imagens de angiotomografias computadorizadas feitas em aparelhos multislice de 16 a 128 canais, sendo as imagens de arquivo pessoal dos organizadores. $O$ atlas promove a identificação das estruturas vasculares em questão e propicia a integração do conhecimento adquirido em salas de aula com a visualização de exames de imagem complementares rotineiros. A indiscutivel presença de exames de imagem adicionais na rotina dos profissionais da saúde atuais faz com que seja válida a estratégia de integração entre o método tradicional de ensino e as práticas ativas de aprendizagem, as quais vêm ganhando destaque recentemente, devido a sua efetividade na fixação de conhecimentos.

Palavras-chave: anatomia seccional, educação médica, tecnologia radiológica. Abstract: Anatomy and Radiology are subjects widely present in health courses at universities. The intersection between Radiological Anatomy and recent technologies, such as interactive teaching platforms, is characterized as a tendency to be followed in the educational sphere, and the implications of this process are not much debated. The Department of Anatomy of Juiz de Fora Federal University has begun the development of an atlas of radiological vascular anatomy, which features printed and interactived versions. It presents computed tomography images taken on multislice devices from 16 to 128 channels, and the images are from the personal authors' archives. The Radiologic Vascular Atlas provides the identification of vascular structures in study and makes easier the integration of knowledge acquired in classrooms with the visualization of routine complementary imaging exams. The indisputable presence of complementary imaging examinations in the routine of current health professionals makes valid the strategy of integration between the traditional teaching method and active learning practices, which have recently gained prominence, due to its effectiveness in setting knowledge.

Keywords: cross-sectional anatomy, medical education, radiologic technology.

\section{Introdução}

Disciplinas clássicas da graduação de Medicina, a Anatomia e a Radiologia possuem inúmeros alicerces em comum, sendo a interdisciplinaridade entre essas áreas proveitosa para o aprendizado do estudante (1). Dessa forma, a Anatomia Radiológica caracteriza-se exatamente pela

Faculdade de Ciências Médicas e da Saúde Juiz de Fora (Suprema/JF), Departamento de Imagenologia, Anatomia I e Anatomia II, Juiz de Fora, MG, Brasil.

2 Universidade Federal de Juiz de Fora (UFJF), Departamento de Anatomia do Instituto de Ciências Biológicas, Juiz de Fora, Minas Gerais, Brasil.

Universidade Federal de Juiz de Fora (UFJF), Juiz de Fora, MG, Brasil.

4 Faculdade de Ciências Médicas e da Saúde de Juiz de Fora (Suprema), Juiz de Fora, MG, Brasil.

5 Universidade Presidente Antônio Carlos (UNIPAC), Faculdade de Medicina, Juiz de Fora, MG, Brasil. 
interseção entre as áreas supracitadas e propicia o estudo da função e das estruturas do corpo por meio de técnicas radiológicas. Nesse sentido, atualmente, a tomografia computadorizada consiste em uma importante ferramenta para detecção de afecções, notadamente devido à introdução da técnica em espiral no início da década de 1990 do século passado, a qual proporcionou excelente análise volumétrica e tridimensional de pequenas estruturas (2, 3). Assim sendo, a integração de tecnologias radiológicas associadas ao aprendizado tradicional da Anatomia surge como interessante ferramenta a ser analisada para aplicação na graduação de estudantes da área da saúde $(4,5)$.

Pesquisas na área de inovação tecnológica educacional aplicada à Anatomia ainda não têm sido extensamente realizadas (6), porém recentes estudos randomizados acerca da inovação tecnológica no ensino da educação médica demonstraram aumento da satisfação, envolvimento e aquisição do conhecimento nos alunos em questão (6). Todavia, muitos anatomistas acreditam que o aprendizado baseado em práticas cadavéricas continua sendo um pré-requisito para o uso de recursos tecnológicos voltados à anatomia, enfatizando a necessidade do ensino nos laboratórios com a gradual incorporação de instrumentos tecnológicos e não a substituição pelos mesmos (7).

\section{Desenvolvimento}

É inegável que, influenciada por fatores como a expectativa social quanto ao atendimento médico, bem como a rápida evolução das ciências médicas e a diversidade de técnicas pedagógicas em discussão, a educação do profissional médico esteja em dinâmica mudança (6). Ademais, o ambiente da saúde, assim como qualquer segmento da sociedade, é constantemente influenciado por fatores gerais, como a globalização e o fácil acesso à internet. Nesse sentido, a educação voltada à integração tecnológica mostra-se vantajosa ao oferecer contextos autênticos para o aprendizado, prática deliberada ao aluno e desenvolvimento na melhora da percepção visual de alterações e de habilidades de detecção (6), além de ser um importante aparato para flexibilizar o estudo da própria anatomia tradicional (8).

Tendo em vista a tendência mundial de aplicação de tecnologias como forma ativa de aprendizado no currículo médico (1, 9), o Departamento de Anatomia da Universidade Federal de Juiz de Fora optou por iniciar produções nesse sentido. Paralelamente ao projeto de treinamento profissional da universidade intitulado "Radiologia aplicada ao ensino da Anatomia", iniciou-se o desenvolvimento de um atlas radiológico vascular de anatomia, o qual inclui versões interativa (Figuras 1 e 2) e impressa

(Figuras 3 e 4). No referido projeto, foram usadas amostras de angiotomografias computadorizadas, sendo as imagens de acervos pessoais dos organizadores e que foram realizadas em aparelhos multislice de 16 a 128 canais, sempre com contraste iodado não iônico e com posteriores reconstruções multiplanares e em três dimensões. A intenção dos autores é de que a publicação do atlas seja uma válida ferramenta para a integração, por parte dos estudantes, do conteúdo aprendido em aulas teóricas e práticas de Anatomia e do reconhecimento das estruturas em exames de imagem rotineiros, aos quais médicos recém-formados estarão em contato permanente, sendo sua interpretação essencial para tomadas corretas de decisões. A referida obra conta com imagens angiotomográficas de todos os segmentos corporais (cabeça e pescoço, tórax, abdome, membros superiores e membros inferiores), e permite, especialmente na versão virtual interativa, que o navegante, ao clicar na estrutura vascular assinalada, destaque-a e evidencie seu nome.

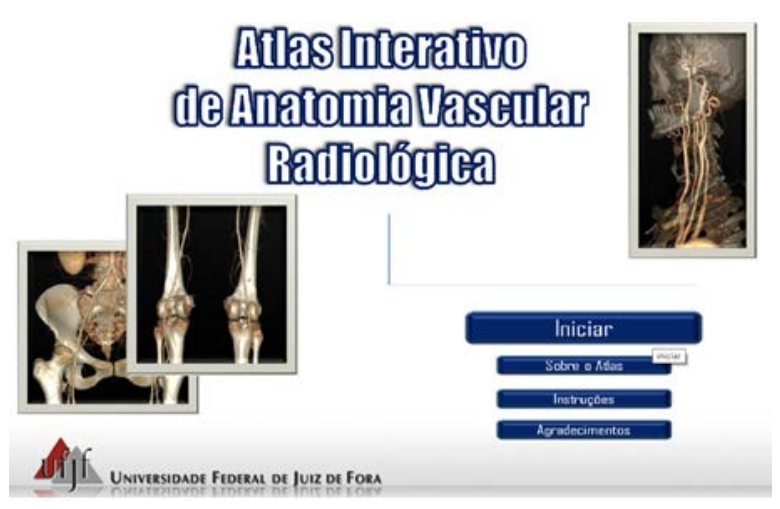

Figura 1 - Menu do atlas virtual interativo. Fonte: Arquivo dos autores. 


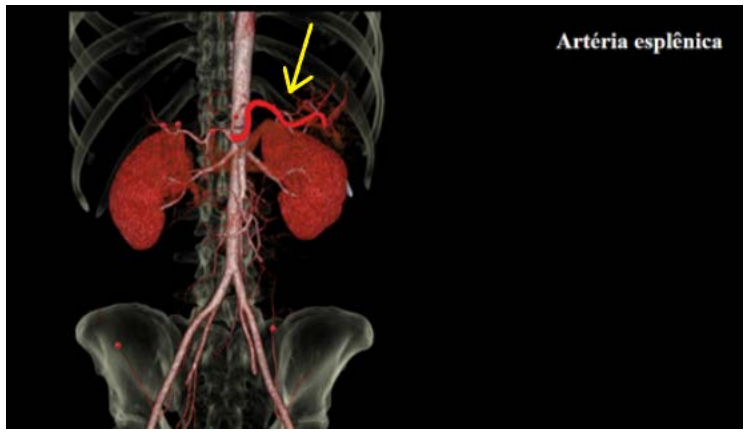

Figura 2 - Figura do atlas interativo, destacando a artéria esplênica. Fonte: Arquivo dos autores.

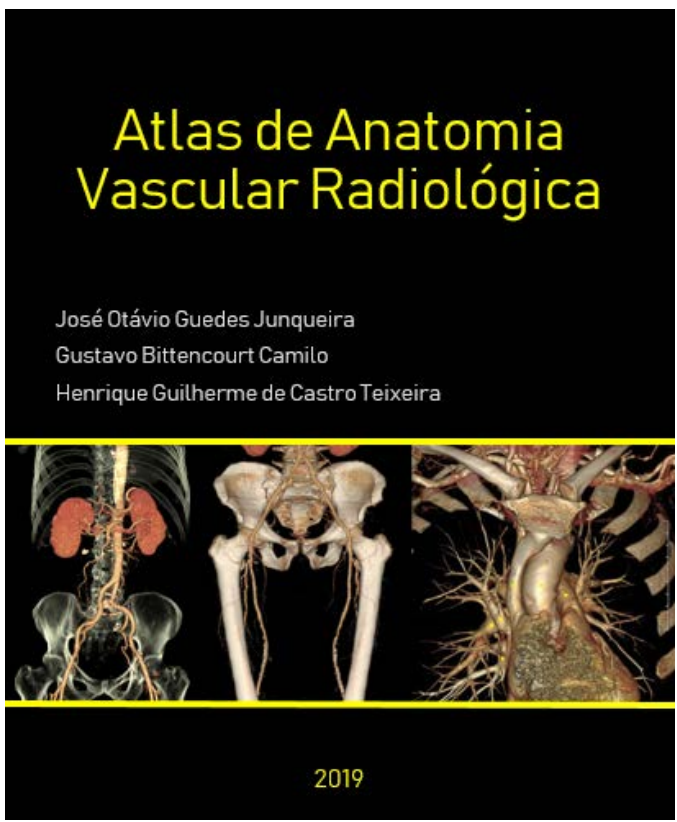

Figura 3 - Capa do atlas impresso. Fonte: Arquivo dos autores.

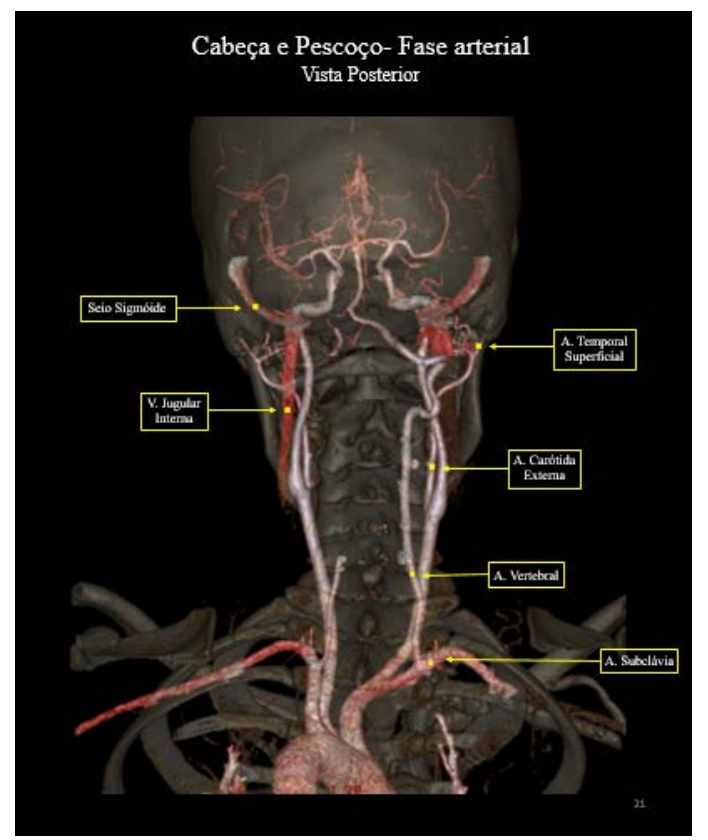

Figura 4 - Figura do atlas impresso. Fonte: Arquivo dos autores.

\section{Discussão}

A onipresença dos exames de imagens no contexto atual da medicina como um todo, demanda investimento e atenção às tecnologias em uso e às inovações desde a formação médica (9), sendo estratégias ativas de aprendizado efetivas propostas para a melhora do poder diagnóstico do futuro médico. Tendo o referido contexto em vista, os autores esperam que o atlas seja um importante aliado na formação, ao passo que auxilia no costume de interpretação de métodos diagnósticos que, por vezes, podem ser pouco discutidos durante os anos de graduação. Um importante objetivo do referido projeto é de que não haja limitações para o acesso do atlas vascular, especialmente em sua versão interativa, a qual se espera que esteja em breve disponivel no site do Departamento de Anatomia da UFJF, constituindo um alcance universal aos estudantes da área médica. Dessa maneira, espera-se que metodologias que aliem a tecnologia na educação médica assumam papel cada vez mais preponderante e essencial no presente e futuro.

\section{Notas}

Parte foi apresentado ao I Congresso de Anatomia Aplicada à Saúde, Juiz de Fora, MG, Brasil, 2019.

\section{Apoio financeiro}

Este estudo não recebeu apoio financeiro de fontes externas.

\section{Declaração de conflito de interesses}

Os autores declaram não haver conflitos de interesses relevantes ao conteúdo deste estudo.

\section{Contribuições dos autores}

Todos os autores fizeram contribuições substanciais para concepção, ou delineamento, ou aquisição, ou análise ou interpretação de dados; e redação do trabalho ou revisão crítica; e aprovação final da versão para publicação. 


\section{Disponibilidade dos dados e} responsabilidade pelos resultados

Todos os autores declaram ter tido total acesso aos dados obtidos e assumem completa responsabilidade pela integridade destes resultados.

\section{Referências}

1. Bahner DP, Goldman E, Way D, Royall NA, Liu YT. The state of ultrasound education in U. S. medical schools. Academic Medicine. 2014; 89(12):1681-6. https://doi. org/10.1097/ACM.0000000000000414

2. Camilo GB, Carvalho ARS, Guimaraes ARM, Kasuki L, Gadelha M, Mogami R, Melo PL, Lopes AJ. Computed tomography airway lumen volumetry in patients with acromegaly: association with growth hormone levels and lung function. Journal of Medical Imaging and Radiation Oncology. 2017; 61(5):591-9. https://doi. org/10.1111/1754-9485.12598

3. Camilo GB, Carvalho ARS, Machado DC, Mogami R, Melo PL, Lopes AJ. CT pulmonary densitovolumetry in patients with acromegaly: a comparison between active disease and controlled disease. British Journal of Radiology. 2015;88(1054):105420150315. https://doi. org/10.1259/bjr.20150315

4. Balogun WG. Using electronic tools and resources to meet the challenges of anatomy education in sub-Saharan Africa. Anat Sci Educ. 2019; 12(1):97-104. https://doi.org/10.1002/ase.1831

5. Yammine K, Violato C. A meta-analysis of the educational effectiveness of three-dimensional visualization technologies in teaching anatomy. Anat Sci Educ. 2014; 8(6):525-38. https://doi.org/10.1002/ase.1510

6. Guze PA. Using technology to meet the challenges of medical education. Trans Am Clin Climatol Assoc. 2015; 126:260-70. PMID: 26330687; PMCID: PMC4530721.

7. Tam M, Hart AR, Williams SM, Holland R, Heylings D, Leinster S. Evaluation of a computer program ('disect') to consolidate anatomy knowledge: a randomised-controlled trial. Med. Teach. 2010; 32(3): (3):e138-42. https://doi.org/10.3109/01421590903144110

8. Estai M, Bunt S. Best teaching practices in anatomy education: A critical review. Annals of Anatomy - Anatomischer Anzeiger. 2016; 208:151-7. https://doi. org/10.1016/j.aanat.2016.02.010

9. Bork F, Stratmann L, Enssle S, Eck U, Navab N, Waschke J, Kugelmann D. The benefits of an augmented reality magic mirror system for integrated radiology teaching in gross anatomy. Anat Sci Educ. 2019; 12(6):585-98. https://doi.org/10.1002/ase.1864

\section{Gustavo Bittencourt Camilo}

Doutor e mestre pelo Programa de Pós-Graduação da Faculdade de Ciências Médicas da Universidade Estadual do Rio de Janeiro (UERJ), no Rio de Janeiro, RJ, Brasil; professor da Faculdade de Ciências Médicas e da Saúde Juiz de Fora - Suprema (FCMS/JF), em Juiz de Fora, MG, Brasil.

\section{José Otávio Guedes Junqueira}

Especialista em Cirurgia Geral pelo Colégio Brasileiro de Cirurgiões (CBC) e pela Associação Médica Brasileira (AMB); professor Adjunto IV do Departamento de Anatomia do Instituto de Ciências Biológicas da Universidade Federal de Juiz de Fora (UFJF), em Juiz de Fora, MG, Brasil.

\section{Henrique Guilherme de Castro Teixeira}

Doutor em Clínica Odontológica pela Universidade Estadual de Campinas (UNICAMP), em Campinas, SP, Brasil; professor titular do Departamento de Anatomia e professor do Curso de Pós-Graduação em Periodontia da Universidade Federal de Juiz de Fora (UFJF), em Juiz de Fora, MG, Brasil.

\section{Alice Belleigoli Resende}

Doutora em Saúde pela Faculdade de Medicina/ Universidade Federal de Juiz de Fora (UFJF), em Juiz de Fora, MG, Brasil; especialista em Educação para as Profissões da Saúde pelo Instituto Regional FAIMER Brasil (Brasil-FRI), Universidade Federal do Ceará (UFC), em Fortaleza, CE, Brasil; professora adjunta do Departamento de Anatomia da Universidade Federal de Juiz de Fora (UFJF), em Juiz de Fora, MG, Brasil.

\section{André Gustavo Fernandes de Oliveira}

Doutor em Neurociências pela Universidade de São Paulo (USP), em São Paulo, SP, Brasil; professor adjunto do Departamento de Anatomia da Universidade Federal de Juiz de Fora (UFJF), em Juiz de Fora, MG, Brasil.

\section{Pedro de Freitas Batista Mendes}

Acadêmico da Faculdade de Medicina da Universidade Federal de Juiz de Fora (UFJF), em Juiz de Fora. MG, Brasil.

\section{Gabriela Cumani Toledo}

Médica Radiologista; mestre em Saúde pela Universidade Federal de Juiz de Fora (UFJF), em Juiz de Fora, MG, Brasil. 
Matheus Cruz Ferraro

Acadêmico da Faculdade de Medicina da Universidade Federal de Juiz de Fora (UFJF), em Juiz de Fora, MG, Brasil.

Thais Bandeira de Oliveira Junqueira

Médica formada na Faculdade de Ciências médicas e da Saúde de Juiz de Fora (Suprema), em Juiz de Fora, MG, Brasil.

\section{Bruna Bandeira de Oliveira Junqueira}

Acadêmica da Faculdade de Medicina da Universidade Presidente Antônio Carlos (UNIPAC), em Juiz de Fora, MG, Brasil.

\section{Endereço para correspondência}

Gustavo Bittencourt Camilo

Faculdade de Ciências Médicas e da Saúde de Juiz de Fora

Alameda Salvaterra, 200

Salvaterra, 36033003

Juiz de Fora, MG, Brasil 